\title{
To Knowledge of the Origin of the Brain Mental Activity
}

\author{
Evgene A. Yumatov \\ Department of Normal Physiology, I. M. Sechenov First Moscow State Medical University, Moscow, Russia \\ Email: eayumatov@mail.ru
}

Received 8 April 2014; revised 1 May 2014; accepted 7 May 2014

Copyright (c) 2014 by author and Scientific Research Publishing Inc.

This work is licensed under the Creative Commons Attribution International License (CC BY). http://creativecommons.org/licenses/by/4.0/

(c) (i) Open Access

\begin{abstract}
This paper is devoted towards the origin of a human subjective state, and we present a methodology for studying the origin of brain activity. We were first to reveal that the subjective states of a human being can be subjected to an objective remote registration. Recently we have established the existence of the so-called "psychogenic field", which reflects the subjective state of human brain. The subjective state of a human being was shown to affect remotely the physicochemical properties of the blood. The interaction of neurophysiological and subjective processes is described in the context of systemic organization of a goal-directed behavior. An original schematic diagram is presented to describe the formation of a subjective brain state. This approach is based on the feedback influence of a psychogenic field on neuronal molecular processes (self-induction in the brain). We propose a paradigm for the origin of a subjective state and possible existence of physical phenomena and brain fields, which are unique for the active brain.
\end{abstract}

\section{Keywords}

Mentality, Consciousness, Subjective State, Brain, Psychogenic Field, Paradigm of a Subjective State Origin

\section{Introduction}

The consciousness, thoughts, senses, and emotions characterize mental activity of the brain, which is manifested in a subjective self-perception and perception of the surrounding world by a human being. The human brain is a unique organization in the living world. The various forms of subjective state can be observed in living beings like sensations, feelings, emotions, consciousness, creativeness, specific language, abstract and heuristic thinking as well self-perception [1]-[4].

K. Popper wrote: "We live in the world of physical bodies and we ourselves are physical bodies. When I speak to you, I do not address your bodies, rather than your minds. Here arises the question of interaction be- 
tween these two worlds, the world of physical states or processes and the world of mental states or processes. This question is a psychophysical problem” [5].

According to S. L. Rubinshtein, there are the following three major viewpoints on this psychophysical problem: psychophysical parallelism, psychophysical interaction, and psychophysical unity. S. L. Rubinshtein held the idea on the unity of mental and physical phenomena, but did not oppose the material to the ideal. "The world is unified, and its unity suggests the materiality; therefore, mental functions should not be separated from the material world" [6].

The matter is an objective reality. There are various forms of matter in the nature. The structural and corpuscular matter has a certain weight. The field matter also exists. The cosmic space really exists and exhibits some properties, e.g., comprises material objects, has a certain length, is curved under the influence of cosmic body gravitation, and affects the distribution of radiations.

The division of brain processes into material and spiritual processes is conditional. We believe that any events which can be revealed and recorded are material. The spiritual phenomena include everything which is perceived inside the body (i.e., in a subjective state). What actually happens is that these processes are united and interrelated in a particular unique organization of the living brain. Hence, the reasoning of a material or nonmaterial nature of the brain's subjective function provides nothing for its understanding.

The appearance of subjective states serves as a major evolutionary factor of living beings, which determines the self-development of life. Subjective states are a strong intrinsic stimulus, which induces active efforts of the body to achieve a specific goal. It characterizes all sides of world perception in the life. These states serve as a directing force in survival and self-preservation of each animal and strain as a whole. Generally the subjective state reflects a strategy vector, which suggests the avoidance of harmful events and achievement of beneficial results.

Initially the subjective states in the brain were manifested as sensations and emotions, which gained the properties of bearings for existing vital demands and their satisfaction. At later stages of brain development, they also comprised mental processes which reflect extrinsic relations in the environment. The reasonable intellectual activity, as a major mean in adaptation and survival, was formed on the basis of this intellectual function of the brain during the follow-up period.

The simplest forms of behavior, including those fixed at the genetic level, are developed with no involvement of a subjective state (unnecessary in this case). These instincts are based on a reflex principle and appear as the actions strictly specified for certain stimuli and conditions.

More complex forms of behavior which suggest the freedom of choice were made possible with the appearance of a subjective state, which provides a strategy of activity under constantly varying conditions. A further evolutionary process could not be realized without the development of a subjective self-perception and perception of the surrounding world. A subjective evaluation of the environment and internal state was a determinant factor of evolution.

A large amount of data exists on the problem of subjective activity of the brain. They include philosophical manuscripts; psychological works describing the exterior forms of mental activity; clinical reports for mental disorders in various diseases; and neurophysiological data on the correlations and mental codes, cognitive, and sensory functions of the brain (e.g., results of functional magnetic resonance tomography [fMRT]). However, the nature (origin) of subjective activity of the brain received little attention. Some authors reported that the subjective nature of brain activity is a mystery of nature, while others believe that this problem is unsolved.

At the present time, there are some indirect methods for recording the psychophysiological state of a human being. They are based on the registration of somatic and autonomic indexes (e.g., BP, ECG, HR, respiratory rate, and galvanic skin response), which reflect the functions of individual organs. At the same time, the subjective and mental processes are not studied under these conditions.

The subjective really exists. In this meaning, the subjective is objective too, though it takes place only in the living organization of matter. The brain activity is dual in nature. On one hand, there are neurophysiologic processes. On the other hand, there are subjective states, characterizing all facets of the world-view [7]-[13].

The neurophysiologic processes can be observed and registered by various methods and devices developed based on laws of physics and chemistry. We do not perceive the processes on the neurophysiologic level, for example, we do not feel the activity of a particular neuron.

The processes on the subjective level are perceived as self-sensations, which cannot be registered directly by physical and chemical methods based on the phenomena in the abiocoen. 
The achievements in modern neurophysiology based on structural, morphological, electrophysiological, neurochemical, molecular, genetic research, computer tomography, etc. do not allow identifying the spiritual functions of the brain. Whatever processes are registered, the electroencephalogram (EEG), neuronal activity, neurochemical and molecular reactions etc., they do not show the internal experiences like thoughts, emotions, senses, etc. The computer tomography of a brain, mapping of gene expression in various brain structures, multichannel record of neuronal activity, multichannel electroencephalogram allow revealing only participation and interaction of various brain structures in the organization of behaviour, training, memory, emotions and thinking. However, these studies do not bring us closer to understanding the origin of subjective states.

The contemporary computer and information technologies allow modeling the central architectonics of behavior [14] in technical devices (robots, automatic control systems). However, we cannot obtain the presence of the subjective: motions, self-perception, etc. It means that the technical organization is not enough to generate the subjective. The brain has a something different that is impossible in the abiocoen.

The brain activity has two facets, visible, characterized by registered neurophysiologic parameters; and hidden or spiritual, manifesting in human subjective perception of oneself and the world around him/her. No doubt, these two facets are interrelated. However, we cannot explain how the code of nerve impulses, the interaction of various brain structures, the electrophysiological processes, the molecular transformations result in the selfperception of subjective state.

In studying the brain, we see only the extrinsic, above-water part of an iceberg; the intrinsic, under-water subjective part is inaccessible for neurophysiological methods.

Functional MRT allows us to perform a vital assessment of the activity and involvement of nearly all brain structures in various forms of mental activity. However, even rigorous analytical studies of the human brain cannot give an explanation on the origin of a subjective state. Even at the highest resolution of functional MRT that provides the recording of activity for each of the billions of brain neurons, it would be impossible for us to understand the origin of a subjective component in brain function.

There are two components in the thought: its subjective nature and its specific content. When researchers write on the possibility to register a subjective state in the neurophysiologic processes, they forget what the subjective is and take the neurophysiologic processes as a real manifestation of the subjective in the brain activity. This means that they do not see any difference between the subjective and neurophysiologic processes and infact identify them.

On the one hand, neurophysiological reactions can be processed to isolate afferent sensory signals for a further image visualization. On the other hand, neurophysiological processes suggest the presence of effector mechanisms for motor, motor-speech, mimic, and autonomic reactions that are induced by the thoughts, emotions, and reminiscences.

There is a huge gap between modern knowledge about brain neurophysiology and understanding of its mental functions. This is due to the fact the brain was studied with the morphological, physical and chemical methods based on the knowledge, phenomena and laws discovered in the investigations of the abiocoen. The origin of a subjective brain state is beyond the field of hi-tech analytical research. This is their essential limitation. There are not even hypothetical, logical concepts in science that explain the origin of the subjective state in neurophysiologic processes.

The objective research of a subjective state calls for different scientific methods and approaches. A living organism and a brain in particular may be the place for physical phenomena and processes that are impossible in the abiocoen. This thesis has principal importance for understanding the essence of the subjective state in the brain activity.

Principally, it is impossible to explain the origin of a subjective brain activity only on the basis of neurophysiological investigations. It is a fundamental delusion when the researchers believe that they guide to the nature of a thought, sense, and other subjective states by studying the electrophysiological processes.

An outstanding psychologist A. N. Leont'ev reasoned that "no direct correlation (comparison) of mental and physiological processes in the brain will solve the problem" [15].

In this regard, N. P. Bekhtereva wrote: “... it is doubtful that the full code of the mental processes will be deciphered only by analyzing the pulse activity of neurons and neuron population. The solution of the task lies no in the sphere of the physiology and biochemistry of a living object, but also in the finest branch of biochemistry: the biology of molecular processes. It is very important to keep reasonable attitude to the material basis of phenomena and to conduct goal-seeking and deeper search towards its deciphering. At the same time, one should try 
to imagine what the ideal is without putting it into "reinforced concrete" bed of materialism? It is noteworthy that sticking to the primitive materialism resulted in that biologists worked in a corridor limited by invisible barbed wire" [16].

When analyzing the problem on the origin of a subjective state, T. Nagel wrote: “... describing mental phenomena, "subjective reality" and coupling them with the neurophysiologic processes faces the "explanation gap" because the mental processes are physical and cannot be referred to spatial and time coordinates. On the other hand, there are no grounds to say that the physical does not accompany the mental, but the question is how? The parallel description of the neurophysiologic processes and mental states caused by them (?) or accompanied by them (?) does not help answering the question how the behavior of a neuron network produces the subjective states, feelings, self-reflection and other phenomena of high order. Without the change of the fundamental concepts of the consciousness, the explanation gap can not be overcome" [17].

The prime objective of psychophysiology is to study the origin of mental processes in brain activity. However, this science is conceptually restricted to the recording of external behavioral and somatic and autonomic manifestations of mental activity, or correlates the involvement and interaction of some brain structures with subjective states.

There is a skeptical opinion among neurophysiologists about studying the subjective activity of the brain. It arises from the fact that the origin of a subjective state cannot be evaluated on the basis of modern neurophysiological approaches. All reasoning's on this subject are nonproductive and do not explain the nature of the subjective state. Moreover, this basic problem is misunderstood and even "factor out" from the area of scientific studies. The subjective brain activity fell outside of scientific researches. This question seems to not exist for neurophysiology. In this regard, a Nobel Prize winner F. Crick et al. told that "among neurophysiologists, all attempts to speak about consciousness were interpreted as the first signs of oncoming senile "dementia" [18].

A way by which the brain generates the inner subjective world remains one of the greatest mysteries in nature. Modern science does not have even hypothetical logic constructions to explain the origin of subjective states in neurophysiological processes.

I. P. Pavlov, R. W. Sperry, P. K. Anokhin, N. P. Bekhtereva, and K. V. Sudakov were optimistic about the possibility of understanding a subjective activity of the brain. They urged us to determine a possible origin of unique brain function, the subjective mental activity [1] [7] [8] [13] [16].

According to I. P. Pavlov, "Marching forward and pressing onward, the natural and unavoidable approach and final fusion of the psychological with the physiological, the subjective with the objective is achieved - the actual question so long disquieting to human thought! And any further facilitation of this fusion is a great goal of the near future in science" [1].

The same thought was expressed by a Nobel Prize winner R. Sperry: "Subjective states and properties, which were driven away from scientific explanation for a long time, should, figuratively speaking, take a driver seat in the theory of brain activity as a crown of evolution" [7].

According to P. K. Anokhin, "specific mechanisms of subjective consciousness generation cannot be described analytically though their exact information relation to the initial parameters of the objective world is beyond doubt" [8]. The subjective state of individuals reflect real psychophysiologic processes [19]. The question that how the brain generates its inner spiritual world remains one of the greatest secrets of the nature.

In our research, we try to find basic approaches to understanding the origin of the subjective in the brain activity, without going into the details of particular subjective state manifestations, consciousness, emotions, etc. This research is based upon the methodological principle that we formulated "the subjective processes can be registered directly only by living structures” [20] [21]. Using this principle, we carried out a variety of series researches, and we for the first time established possibility of direct remote registration of a subjective condition of humans with the aid of the subjective state indicator [20]-[22].

The findings suggest that a human subjective state can be registered objectively and remotely. It is important to note that a subjective state indicator shows his/her subjective attitude towards somebody or something.

The effect, which we found, characterizes specific abilities of individuals. It was found that about $10 \%$ of humans show their own subjective state in subjective state indicator. It is not surprising as everything is unique in the field of biology, from a unique DNA to diversity of individual forms of behavior and display of emotions.

While the credibility of our findings does not raise doubts, we wanted nevertheless to have additional evidences of a direct objective registration of, in blind tests, eliminating skeptical attitude towards our findings. 
In our research, we hypothesized that the human subjective state may manifest itself in different forms. For registration of a human subjective state, we used blood as a biological multicomponent substrate containing cell elements, protein-colloid and electrolyte solutions.

This series was designed to evaluate whether the subjective state of an investigator can remotely and directly affect the blood, specifically the erythrocyte sedimentation rate [ESR] and blood $\mathrm{pH}$.

\section{Materials, Methods and Results}

The Remote Influence of the Human Mental Activity of the Brain on the Physical and Chemical Properties of the Blood.

In these series of tests we try to find the remote direct influence of the investigators' subjective state on blood in particular and erythrocyte sedimentation rate (ESR) in general.

We use blood samples taken from healthy individuals in clinical settings. ESR was measured using standard Panchenkova method. We compared ESR figures in three capillaries fixed in separate test tube racks.

In the first rack, we put a capillary with blood unexposed to any subjective influence (control). In the second rack, we put a capillary with blood approached by the investigator in a neutral subjective state holding the subjective state indicator in his hands (neutral). The pointers of the subjective state indicator remained in the initial position and were directed forward. The neutral control was necessary to exclude any possible outside influence of the investigator on ESR, e.g. associated with movement and approach of the investigators to the rack, with heat, electromagnetic and electrostatic influence of the investigator's clothes, etc.

The third rack with blood capillary was approached by the investigator in a certain subjective state indicated by the crossed position of the pointers (subjective). The subjective state was provoked by the investigator with one of the tests described above [20]-[23], an expressed imagined emotional or cogitative condition.

The subjective state indicator pointers demonstrated the subjective state of the investigator when he/she approached to a blood filled capillary. The investigator perceived his/her subjective state without indicators.

In the tests, the investigator approached the second rack five times in a row (neutral), and then 5 times in a row to the third rack (subjective) with several seconds interval. In separate tests, the number of approaches varied from one to ten. All three tests were performed at the same time with samples taken from one person. All racks were put in vertical position simultaneously. The ESR was calculated in one hour.

We compared the ESR figures in three different capillaries: control, neutral and subjective [24]. The findings were processed with standard mathematical methods to reveal regularity.

The findings demonstrate reliable difference of ESR in control, neutral and subjective tests (Figure 1). All subjective tests showed significant decrease of ESR. The subjective state caused ESR decrease from $1.5 \mathrm{~mm}$ to

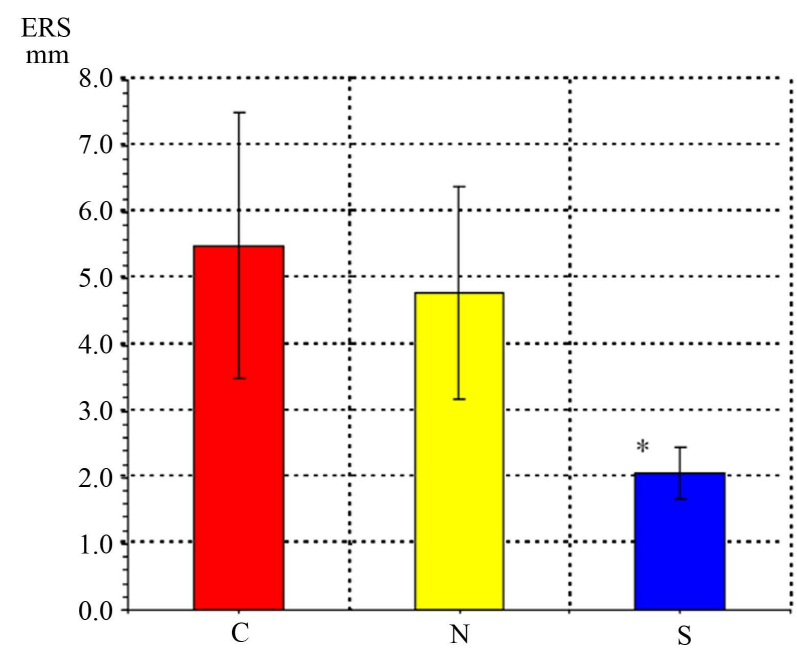

Figure 1. The change of erythrocytes sedimentation rate (ESR) in blood after the remote influence of the investigator subjective state. ERS mm. $\mathbf{C}$ - control, $\mathbf{N}$-neutral, $\mathbf{S}$ - subjective. Reliable difference ${ }^{*} \mathrm{p} \leq 0.05$ between $\mathbf{C}, \mathbf{N}$ and $\mathbf{S}$. 
$2.5 \mathrm{~mm}$. The higher was the baseline ESR figure in the control test of a particular individual, the bigger was the difference between ESR figures in a control and subjective tests. However, no reliable difference was found between the control and neutral tests which suggest that the blood was not affected by the neutral subjective state of an investigator.

Therefore, there arised naturally a question, what is the distance on which a subjective state of the investigator influences on ESR of the blood? We have found that influence of a subjective state of the investigator on blood completely disappears by the removal of the investigator on $0.8-1 \mathrm{~m}$ from the capillary with blood. Thus, the field remote effect depends on distance between the investigator and blood.

Our studies did not reveal a change in ESR upon the influence of a certain subjective state of the investigator on the patient's blood, blood of the investigator, and rat blood.

We were first to provide evidence that a subjective state of the human brain has a strong effect on physicochemical properties of the blood [24].

In the next series, we evaluated the remote influence of a certain subjective state of the investigator on human blood $\mathrm{pH}$. The study was performed with a Mettler Toledo digital $\mathrm{pH}$ meter (measurement accuracy $0.01 \mathrm{pH} \mathrm{U}$ ).

The donor blood (3 - $6 \mathrm{ml}$ ) was obtained from healthy volunteers (men, 25 - 35 years) at the Blood Center of the I. M. Sechenov First Moscow State Medical University. Blood samples were treated with EDTA as an anticoagulant.

$\mathrm{pH}$ was measured in two glasses (treatment glass and control glass). The donor blood (1 ml) from a healthy man was added to Rodnik bottled water $(100 \mathrm{ml})$. The hemolyzed blood specimen was diluted in water and evenly divided between two glasses.

The first glass (treatment group) with hemolyzed blood was approached by the investigator in a certain subjective state. The hemolyzed blood-filled glass was exposed to 1 - 8 sessions of a subjective influence (treatment).

The second glass (control group) with hemolyzed blood was not subjected to any influence. $\mathrm{pH}$ values in the treatment and control glasses were measured consecutively (five-to-twenty times) after each subjective influence. Hemolyzed blood temperature in the glasses was measured simultaneously. The results were analyzed by Student's test (Excel software).

$\mathrm{pH}$ of water was estimated first. Control experiments revealed that a subjective state of the investigator has no effect on water $\mathrm{pH}$.

The human subjective state had a strong effect on hemolyzed blood $\mathrm{pH}$ under conditions of a remote (contactless) exposure. In this case, $\mathrm{pH}$ of the blood was shifted toward alkaline (Figure 2).

The differences between hemolyzed blood $\mathrm{pH}$ in the treatment and control glasses became more significant with an increase in the number of subjective influences.

A remote influence of the human subjective state on $\mathrm{pH}$ values was not observed after dilution of the blood in distilled water, saline, and isotonic glucose solution.

These data prove the existence of a remote-field effect produced by the human subjective state.

\section{Discussion}

\section{A Hypothesis on the Nature of Brain Mental Activity.}

The remote impact of the subjective state can be originated by the field generated by the biological object by the human brain itself. We call this a "psychogenic field" since it reflects the psychic subjective state of a human [21]-[23].

The presence of any field is proved by its impact on processes or physical bodies. The existence of a psychogenic field is revealed by the remote mechanical impact on the pointers indicating various subjective states of a human [22] [25] [26]. Another manifestation of the psychogenic field is its reliable remote impact on ESR and $\mathrm{pH}$. This field may have other properties that we do not know yet. The remote manifestation of the psychogenic field depends upon its strength and spatial orientation, which may account for individual differences.

These findings pose question about the origin of psychogenic field, about the substrate that generates it, and about biological infrastructures it affects? These questions necessitate further research.

Historically the biological science developed based on the knowledge and achievements of abiocoen physics. As for the physical laws of living beings, these laws were overlooked by the traditional physicists and biologists. Researches into the subjective human brain states, we for the first time approached the physical field processes that are possible only in living organisms. No technical means are capable to register spiritual state. Subjective states can be perceived only by living structures. 


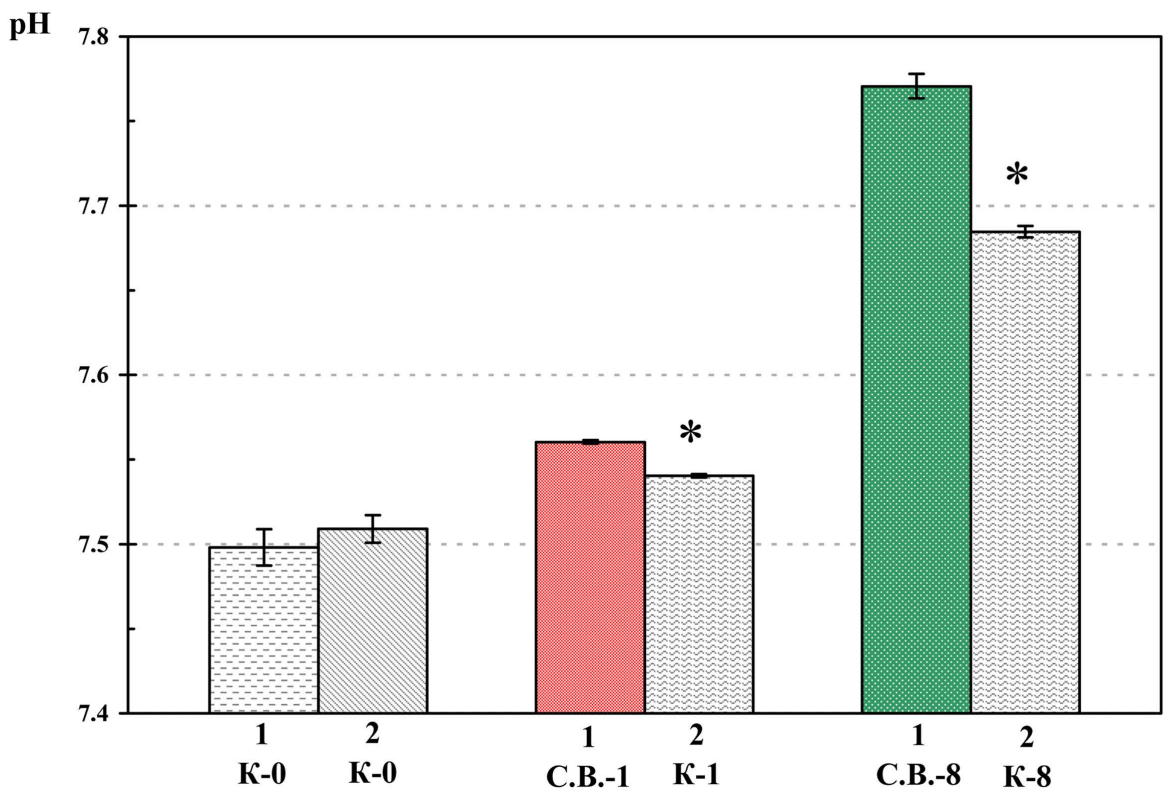

\begin{abstract}
Figure 2. Remote influence of a certain human subjective state on blood $\mathrm{pH}$. Designations: $\mathrm{pH}$ measurements in glass: K-0 (initial pH of blood in the first (1) and second (2) glasses, no subjective influence). After a single (C.B.-1) and 8-fold exposure to subjective influence (C.B.-8). Treatment glass $\mathbf{- 1}$ (remote influence of a subjective state); control glass- $\mathbf{2}(\mathbf{K}-\mathbf{1}$, and $\mathbf{K}-\mathbf{8}$ no subjective influence). Temperature varies from $24.3^{\circ} \mathrm{C}$ to $24.5^{\circ} \mathrm{C}$. $\mathrm{N}$, number of $\mathrm{pH}$ measurements (60). The significance of differences $\left({ }^{*}\right)$ between C.B.-1 and $\mathbf{K - 1}$, as well as between C.B.-8 and $\mathbf{K}-8$, is $\mathrm{p}<0.01$.
\end{abstract}

We see that the psychogenic field really exists; reflects the subjective state of a human, associated with the brain activity; causes forces affecting on the subjective state indicator; produces remote impact on the physical properties of blood; and therefore is of physical nature.

In the field of subjective brain activity, the major question arises: how the brain, which serves as a unique living structure, can in principle generate the subjective state or self-sensation? In other words, which should be the psychophysical organization to reproduce a subjective attitude to itself?

In literature there are a lot of works on the problem of subjective human brain activities. This includes philosophical articles; psychological in which external forms of mental activity are described; the clinical works considering violation of mental functions at various diseases; neurophysiological in which authors try to reveal correlates and codes of mental cognitive, sensory functions of a brain including by means of fMRT. However among them there are no articles on experimental studying of the nature (origin) of subjective brain activities. A number of researchers note that the subjective aspect of brain activities remains to be one of secrets of the nature, and some of them consider that this problem is still unsolved.

In other words, it is impossible to analyze the origin of the subjective basing on the contemporary neurophysiologic methods, and all speculations are counterproductive and are unrelated to the understanding of the nature of the subjective state.

Several researches inspired by the achievements in neurophysiology, molecular biology, nanotechnologies, predict the possibility of deciphering of "thinking codes", consciousness and image visualization of neuron pulse activity [12] [27]. In fact, it is possible in some cases to see certain correlation between the activity of certain brain structures or neurons and the content side of thinking, speech, etc. This however does not mean that in these processes, one can understand the subjective state.

Among the billions of brain neurons, one can always find neurons whose activity will correlate with a particular function of the body. At the same time, the information encoding may differ among individuals and social groups. Each neuron registered in the brain is individual and different from the others. The investigator will never find this neuron in this or any other individual.

S. Hameroff hypothetically connects emergence of subjective human brain activities with structure and function of neurons microtubules. The author suggests that subjective state of a brain arises at quantum level of its 
organization, and the brain is the quantum computer. According to the author "brain processes relevant to consciousness extend downward within neurons to the level of cytoskeletal microtubules. An explanation for conscious experience requires (in addition to neuroscience and psychology) a modern form of pan-protopsychism in which proto-conscious quails are embedded in the basic level of reality, as described by modern physics" [28].

However these theoretical views aren't supported with pilot studies and the evidence of their communication with subjective brain activities isn't produced. They don't allow to understand, how the brain, unlike computers and other lifeless systems, creates the internal subjective state, i.e. itself feels?

To understand the nature of a subjective state, it is necessary to imagine a principal circuit of the brain organization that can reproduce subjective self-perception.

We believe that this brain organization should generate specific field processes in the molecular structure, which produce a secondary effect on its neurophysiological mechanisms.

It is a well known fact that the electromagnetic field has a reverse effect on the generation and conduction of excitation in excitable cells (e.g., in neurons, nerves, and muscles). Using the neuron or muscle as an example, it can be shown that primary membrane ion fluxes generate the action potential (synaptic potential). Due to the propagating field (electrotonic effect), they affect the adjacent electrically excitable structures and cause a progressive propagation of excitation. The electrotonic field influence can modulate nerve cell excitability.

Taking into account the notions on physical self-induction (Lents, E. Kh., 1833; Faraday, M., 1834), data on the electrotonic effect of field on excitable cells (Tasaki, I., 1957), and results of our experiment, we proposed an original scheme for the subjective brain state (Figure 3 and Figure 4), which is based on the closed-loop field effects.

The electric physiology of excitable structures suggests that the electromagnetic field generated by a nerve cell affects the excitability and can cause excitation and conduction of a nerve pulse. We think that the fields generated by the brain produce reverse influence on the neurophysiologic mechanisms of the brain.

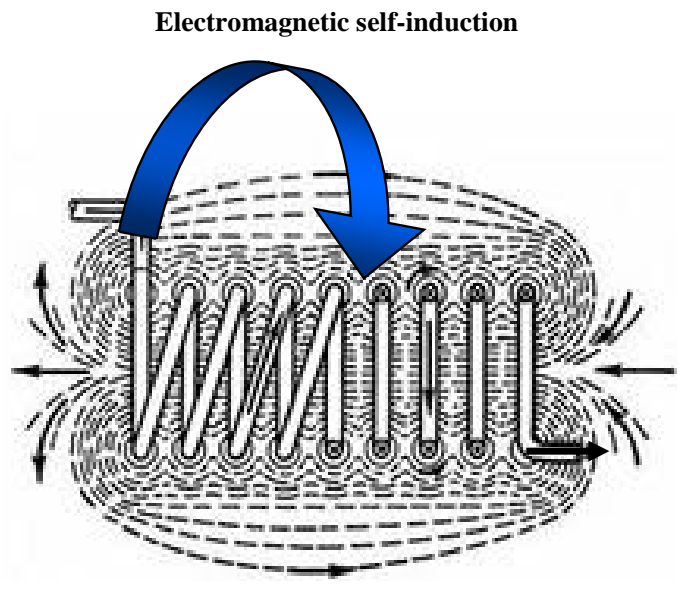

Lents, E. Kh., 1833; Faraday, M., 1834

Field electrotonus

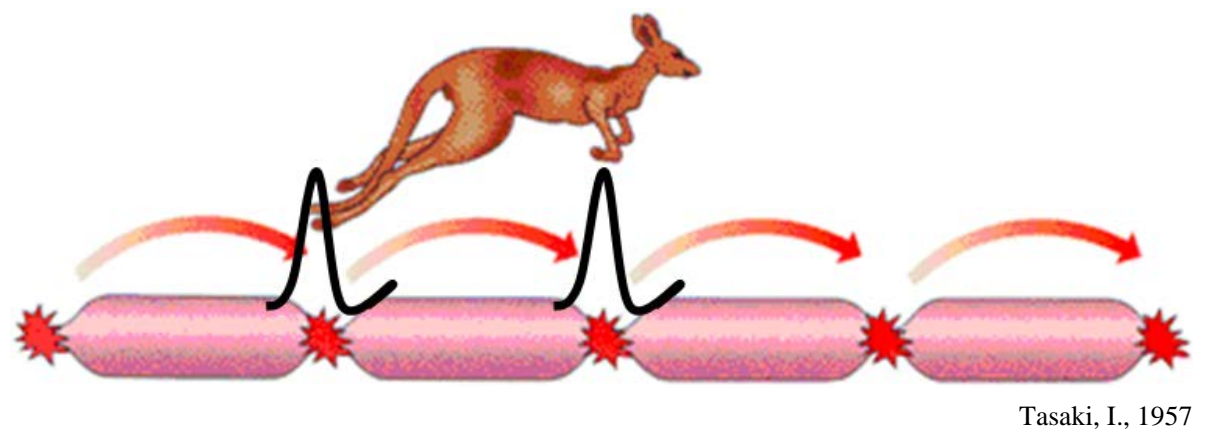

Figure 3. Closed-loop field effects. The signal is transmitted very quickly (400 km/h). Normal nerve, the myelin sheath is not injured. 


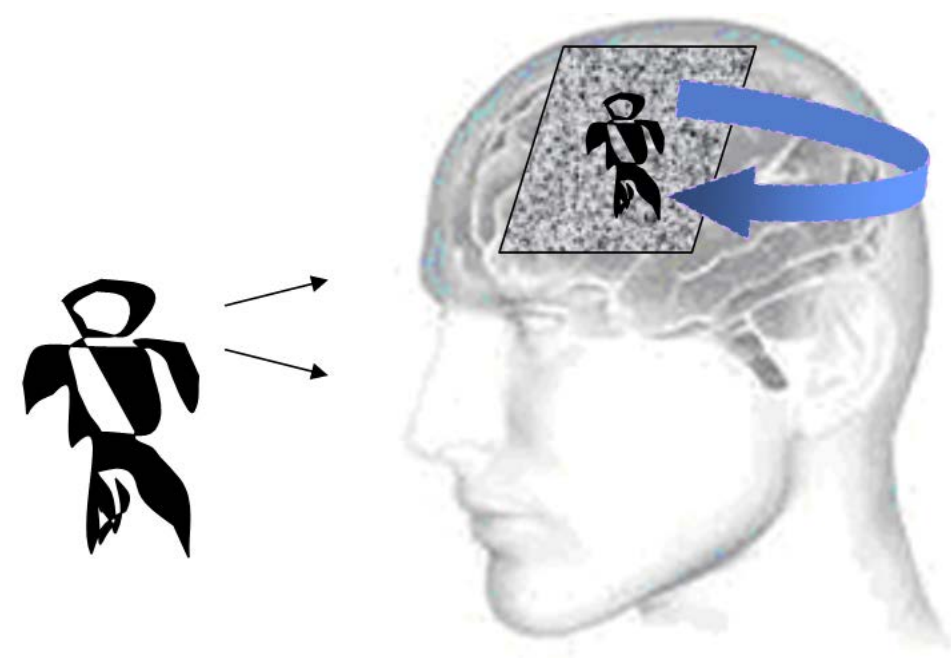

Figure 4. Self-induction in the brain. Hypothetical principal scheme for the formation of a subjective brain state. Reverse effect of the psychogenic field on neuronal molecular processes.

These processes illustrate a "reverse effect" of the field generated by nerve tissue. In our opinion the brainderived psychogenic field has a secondary reverse effect on neuronal molecular processes, which contributes to a subjective state of the whole brain. Neuronal structures of the brain serve not only as a "generator" of the specific brain field, but also as a target "screen" for this field. These data characterize a closed loop of self-perception processes in neuronal structures of the brain.

Following the physical analogy we can call the reverse influence of the field on the structural and molecular processes in the brain as "brain self-induction".

Our future studies will be designed to answer the following questions. First, which molecular structures of the brain can serve as a source of the psychogenic field? And second, which molecular structures can perceive this field?

The Neurophysiologic and Subjective Processes in the Functional System of Goal-Seeking Behavior.

The theory of functional systems developed by P. K. Anokhin [14] and presented in the works of K. V. Sudakov [19] and many other researchers points at the main mechanisms in the brain activity, which may be associated with origin of emotions and thinking. However the central architecture of a behavioral act reflects only neurophysiologic component and fails to represent the organization of the subjective processes.

The contemporary concept of the system organization of goal-seeking behavior lacks the separate description of processes taking place on the neurophysiologic and subjective levels. The subjective is left "off-camera" and it is only presumed existing.

It is quite understandable that this situation is because there was no possibility to register objectively the manifestation of the subjective state in the brain.

In the system organization of a goal-seeking behavior, we split the brain functions taking place in the neurophysiologic and subjective levels and presented the interaction of the neurophysiologic and subjective processes in Figure 5.

All known neurophysiologic phenomena registered in the brain can be associated with various main mechanisms of functional system of goal-seeking behavior [14] [19]. In addition to that, there are processes in the functional system of goal-seeking behavior, which take place only on the subjective level and without which the brain activity is impossible.

The functional system of goal-seeking behavior has two interrelated levels of brain organization: neurophysiologic and subjective. The system processes take place both in the neurophysiologic and subjective spheres of brain activity.

All processes in the brain start at the neurophysiologic level and then develop on the subjective level, which is a kind of a "hadow" of the neurophysiologic processes. A. M. Ivanitsky showed that a sensor stimulus produces a neurophysiologic response followed by a subjective sensation [10] [11]. 
2. The subjective level

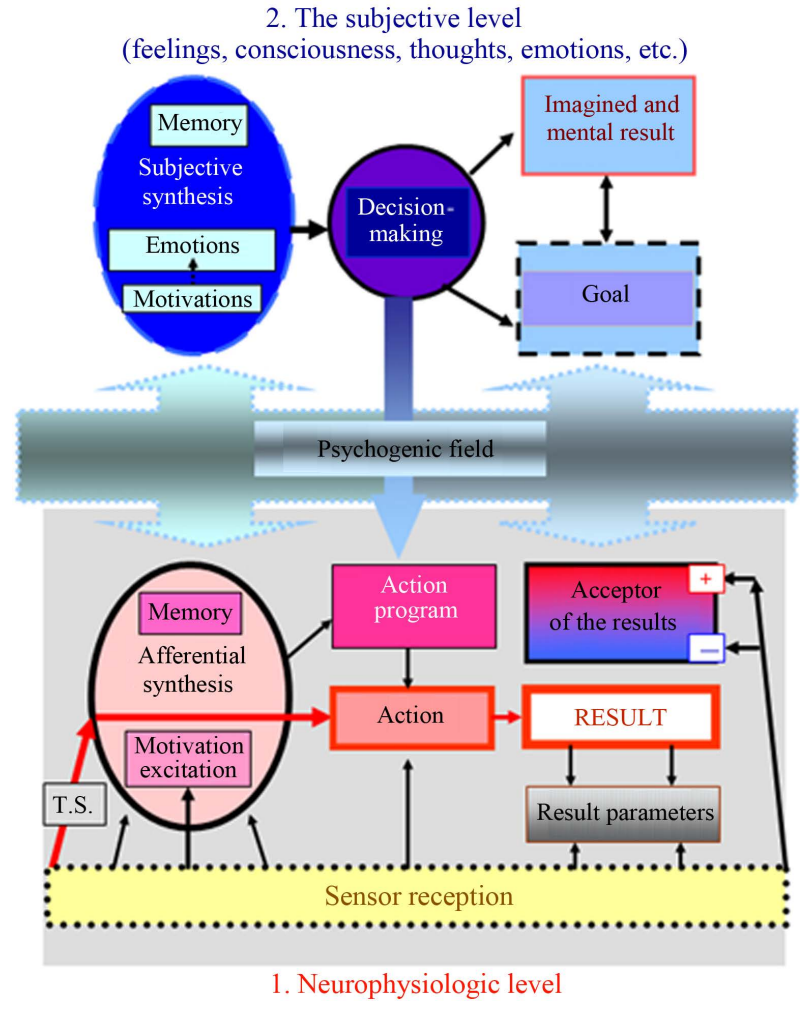

Figure 5. The hypothetical schematic drawing of the functional system in the context of the "goal-seeking" behavior: interaction of neurophysiologic (1) and subjective (2) processes in the brain activity. Abbreviations used in figure: TStrigger stimulus.

At the first stage, the afferential synthesis goes on the neurophysiologic level, and then the process of analysis and interpretation continues on the subjective level_- “subjective synthesis”, including the sensation of attraction (motivation), situational assessment, memorization and recall. The process is finished by decision-making and goal-setting made on the subjective level.

The further development of the processes in the brain may follow two ways.

In one case, the subjective synthesis finishes with the acceptance of an imagined mental result in the framework of subjective mental functional system, the existence of which was highlighted by K. V. Sudakov [13] [19].

In the other case, the decision about the goal-seeking behavior and goal-setting returns the processes from the subjective sphere to neurophysiologic level to formulate the program of result-oriented behavior and action results acceptors, in which the parameters of the future result is prognoses in accordance with the set goal.

Then, in the neurophysiologic level framework, everything develops according to the known scheme: an action is formed aimed at the achievement of a necessary result, parameters are registered, and through the reversed afferentation channels, these parameters go to the acceptor to be compared with parameters of the prognoses result.

If the prognoses and achieved results match, the process is stopped and a new stage of goal-seeking behavior without the participation of subjective level of the functional system is started.

If the prognoses and achieved results match, the result acceptor initiates a "mismatch" or "surprise" reaction. The process goes to the subjective level where negative or positive emotion is generated depending upon the failure or no-failure to achieve the goal.

The positive emotion produces satisfaction and fixation finishing a specific behavioral act. The negative emotion mobilizes the process of subjective synthesis to search another more appropriate decision that will allow achieving the goal.

The emotions are the means of memory fixation of the whole bunch of factors promoting or hindering 
achieving the goal. At the neurophysiologic level, new emotions are transformed into emotional reactions of the body. The behavior is oriented along the common vector from a negative reaction to a positive one.

The emotional reactions are formed in the neurophysiologic part of the functional system, while the emotions and thoughts are produced in the subjective part. A thought reflects the informational content of the prognosis and necessary result and emotions reflect the probability characteristic of possibility and feasibility to achieve the goal.

Memory has the following two components: the neurophysiological component, and the subjective component. The process of memorization occurs at the neurophysiological level, which involves subjective activity of the brain. The process of data storage in memory proceeds at the neurophysiological level with no involvement of subjective perception. Reminiscence and information retrieval from memory always involve subjective (intellectual) activity of the brain.

In essence, modern studies of memory are limited to the neurophysiological mechanisms. The mechanisms of memory cannot be evaluated without studying the subjective sphere. Hence, the process of information retrieval from memory is yet unknown.

The structural and neurophysiological component of the functional system cannot realize a resultant activity without the subjective environment. In a similar way, the subjective subsystem is formed on the basis of neurophysiological processes.

The neurophysiological level serves as a basis for various processes in the body, including the perception of sensory excitation signals from the sense organs; formation of biological motivations, components of the memory associated with remembering and storage of information, and efferent command programs for the regulation of movements and behavior; realization of all somatic and autonomic reactions; and evaluation of the achieved result.

At the neurophysiologic level, the tailored reflex reaction and automatic behavioral acts are performed due to preoperational integration, which is formed earlier [14]. In these cases, the behavioral choice takes place without the participation of consciousness, and the interpretation may follow the action if the prognoses result was not achieved, or the interpretation may be omitted if a certain result is achieved.

The neurophysiologic level lies in the basis of perception of the environment and the internal state of the body, of organization of various behavioral forms and the body vital functions regulation.

An understanding of incoming information in the brain, manifestation of social motivations, initiation of information retrieval from memory, formation of the goal, and development of all psychological states (consciousness, thinking, emotions, etc.) are realized at the subjective level. The major mental functions of the brain, including the freedom of will, goal-setting activity, choice of the behavioral strategy, expected (imagined) result, and assessment of goal achievement, occur at the subjective conscious level.

The freedom of will, behavioral choices, imagined result and assessment of goal achievement take place at the subjective conscious level.

Our notion of consciousness arises from the aforesaid. Consciousness is a subjective brain activity, which reflects the inner state of the body and environment and forms a basis for goal setting, decision making, prediction, and evaluation of the action result. The consciousness includes emotions, feelings and thoughts that eventually determine the freedom of choice, decision-making and the result assessment.

What is the biological significance of evolutionary development and existence of subjective world perception? Why did the organism serve as a pioneer of the subjective in living nature? These questions can be answered as follows.

The assessment of a biological and/or social significance of incoming information from the sense organs is realized via the subjective state. The subjective state contributes to the choice of an effective goal-directed activity, which results in the satisfaction of biologically or socially important motivations. The living organism selects a "target" under constantly varying conditions, in different probability situations, and among a variety of behavioral reactions. The subjective state is a strong internal stimulus for action to achieve the goal.

As differentiated from living organisms, complex technical devices have the target (purpose) that is preset by a designer. The conditions to achieve this purpose are strictly determined and limited.

During the self-development of life, the presence of a subjective state is essential for evolution and existence of higher living organisms.

Decision-making and behavioral choices are always determined. The feeling of unrestricted freedom and the independence of choice are illusion, i.e. self-perception in one's consciousness. If we observe all the possibilities 
of an individual's actions, we see in fact that the individual had several possibilities and chose one. If we could see the processes in the individual's brain we would see that the choice was determined and the only possible basin on the combination and layout of all components of subjective and objective processes at a given moment of time.

It is not important for the individual what the freedom choice is: an illusion or really one's choice. Subjectively, he/she perceives this process as his/her decision. The freedom of choice is influenced by education, schooling, culture, etc., which form the perception of moral, laws, crimes, etc. That is why an individual bears the personal responsibility.

The subjective spiritual state of the brain results from the interaction and interrelation of neurophysiologic processes and brain-specific fields. Subjective and objective processes in the brain are closely interrelated and this relation is dual. This is a unique feature of the brain as a living organization of matter in the existing world.

The nature of a subjective ideal state is in the objective processes of the brain activity. The subjective is a phenomenon, function, state of brain, which, we think, emerges in the interaction of structural and molecular (neurophysiologic) and field processes in a living brain.

The origin of a subjective brain state is in the fundamental properties of a living brain, which is a specific type of matter and has its own physical laws and specific brain fields.

We think that one should not identify the notions of the information and the subjective. The information is present both in inanimate and animate objects. The notion of information does not define the nature of and is not an analog of the subjective. The information should not be viewed as an ideal substance above the real processes. The information is a link function among the elements of the system [22].

The central architectonics of the goal-seeking behavior functional system consists of two interrelated and united subsystems: a structural and neurophysiologic one and a subjective one. The structural and neurophysiologic component of the functional system cannot perform an effective activity without the participation of the subjective sphere, and the subjective subsystem is formed based on neurophysiologic processes.

The research into the neurologic, mental diseases is often limited to the objective neurophysiologic processes thus the researchers want to see them as the cause of the brain functional disorders. However, the origin of many brain diseases is in the subjective sphere of brain activity, which indirectly manifests themselves in the neurophysiologic processes. This may be the explanation of the unsolvable problems in the understanding of etiology and pathogenesis of many psycho neurologic disorders.

The subjective state of a human has independent significance: it determines the recovery, treatment efficiency, disease outcome, and the successful psychotherapy is associated with the pact on the subjective sphere.

\section{Conclusions}

The Paradigm of the "Subjective": basic postulates.

The world-known expert in the field of scientific methodology T. Kuhn wrote: "The decision to decline a paradigm is simultaneously a decision to take another paradigm, and the sentence bringing us to this solution includes both comparison of both paradigms with nature and the comparison of the paradigms to each other [29]. The science lacks any paradigm about the origin of a subjective state besides common view that a subjective state is in a way originated in the systemic brain mechanisms and neurophysiologic processes.

For the organization of brain activity systemic and basing on our tests of human subjective state registration, we came to the basic postulates given below, which characterize interrelation of subjective and objective processes in a human brain.

- The origins of a subjective state are in basic properties of the living brain, which serves as a particular form of matter and has the proper physical laws and specific brain fields.

- The functional system of goal-directed behavior has two interrelated levels of brain organization (neurophysiological level and subjective level) and represents a united integral systemic structure.

- The subjective state of the brain results from interaction and interrelation of neurophysiologic processes and brain-specific biological fields ("psychogenic field").

- The subjective states can be perceived only by living structures.

- The biological fields produced by the brain can directly affect the structural and functional processes in the brain ("biological self induction").

- Psycho neurologic diseases can primarily occur in the subjective processes and secondarily manifest them- 
selves in various structural and functional disorders.

- Living brain physics is a new field of science analyzing unique physical phenomena characteristic only to a living brain and non-existent in abiocoen.

\section{References}

[1] Pavlov, I.P. (1951) The Twenty Years of Objective Study of Animal Higher Nervous Activity (Behavior). In: Pavlov, I.P., Ed., Complete Compilation of Works, The Publishing House of the Academy of Sciences of the USSR, Leningrad, 3, 392 p. (in Russian).

[2] Damasio, A. (2000) The Feeling of What Happens: Body and Emotion the Making of Consciousness. Harcourt Brace, New York.

[3] Edelman, G. and Tononi, G. (2000) Consciousness. How Matter Becomes Imagination. Penguin Books, London.

[4] Reber, A. and Reber, E. (2001) Dictionary of Psychology. 3rd Edition, Penguin/Viking, London.

[5] Popper, K. (2008) Knowledge and Psychophysical Problem. In: Zhuravlyov, I.V., Ed., Protection of Interaction, Lietuvių Kalbos Institutas, Moscow, 256 p. (in Russian)

[6] Rubenstein, S. L. (2003) Life and Consciousness. People and World, Moscow. (In Russian)

[7] Sperry, R.W. (1952) Neurology and the Mind-Brain Problem. The Journal of American Science, 40, $291-312$.

[8] Anokhin, P.K. (1969) The Psychic for of the Reality Reflection. In: Pavlov, T., Ed., Lenin's Theory of Reflection, Science and Art, Sofia, 109. (in Russian)

[9] Bekhtereva, N.P., Budsen, P.V. and Gogolotsin, Y.L. (1977) Brain Codes of Psychic Activity. Science, Leningrad. (in Russian)

[10] Ivanitsky, A.M. (1999) The Main Secret of Nature: How Brain Processes Result in Subjective Experience. Psychological Journal, 20, 93-104. (in Russian)

[11] Ivanitsky, A.M. (2005) Consciousness and Brain. Journal in the World of Science, 11, 1-8. (in Russian)

[12] Koch, C. (2005) Neurobiology of Consciousness. MIT Press, Cambridge.

[13] Sudakov, K.V. (2010) System Mechanisms of Psychic Activity. Korsakov Journal of Neurology and Psychiatry, 110, 4-14. (in Russian)

[14] Anokhin, P.K. (1968) The Biology and Neurophysiology of Conditional Reflex. Medicine, Moscow. (in Russian)

[15] Leont'ev, A.N. (1975) Activity, Consciousness, Personality. Politizdat, Moscow. (In Russian)

[16] Bekhtereva, N.P. (1990) Per aspera... Life. Science about a Brain of the Human. Science, Leningrad. (in Russian)

[17] Nagel, T. (2001) Think Ability of Impossible and Problem of a Spirit and a Body. Philosophy Questions, 10, 92-107. (in Russian)

[18] Crick, F. and Koch, C. (1995) Why Neuroscience May Be Able to Explain Consiousness. Scientific American, $273,66$.

[19] Sudakov, K.V. (2010) On the Theory of the Unity of the Material and Ideal in the Human Activity. Journal of Human, 6, 5-16. (in Russian)

[20] Yumatov, E.A. (2010) Information and Field Effects of Registration of the Human Brain Subjective State. Clinical Informatics and Telemedicine, 6, 98-104. (in Russian).

[21] Yumatov, E.A. (2010) The Direct Registration of a Human Subjective State. Bulletin of New Medical Technologies, 4, 187-192. (in Russian)

[22] Yumatov, E.A. (2011) System Psychophysiology of Human Subjective State. Sputnik +, Moscow. (in Russian)

[23] Yumatov, E.A. (2011) The Physics of Human Brain Subjective State. Consciousness and Physical Reality, 10, 39-50. (in Russian)

[24] Yumatov, E.A., Bykova, E.V. and Dzhafarov, R.N. (2013) Long-Distance Effects of Human Subjective Status on the Blood Physicochemical Characteristics. Bulletin of Experimental Biology and Medicine, 155, 527-530.

[25] Yumatov, E.A., Bykova, E.V., Dzhafarov, R.N. and Tsarenko, O.I. (2013) The Proof Remotely-Field Implication of a Subjective State of a Brain of a Human. Consciousness and Physical Reality, 2, 33-38. (In Russian)

[26] Yumatov, E.A. (2012) About the Nature Subjective in Activity of a Brain of the Person. Russian Medicobiological Bulletin, 2, 116-131. (in Russian)

[27] Anokhin, K.V. (2010) Lecture on the Brain Codes. (in Russian) http://nature-wonder.livejournal.com/175914.html

[28] Hameroff, S. (2007) Consciousness, Neurobiology and Quantum Mechanics: The Case for a Connection. In: Tuszynski, J., Ed., The Emerging Physics of Consciousness, Springer-Verlag, Berlin, 193-253.

[29] Kuhn, T. (1962) The Structure of Scientific Revolutions. University of Chicago Press, Chicago. 